\title{
Feasibility of Electrospraying Fully Aqueous Bovine Serum Albumin Solutions
}

\author{
Maarten Batens $^{\mathrm{a}, 1}$, Loís Dewaele ${ }^{\mathrm{a}}$, Jan Massant ${ }^{\mathrm{b}}$, Bianca Teodorescu ${ }^{\mathrm{c}}$, \\ Christian Clasen ${ }^{\mathrm{d}, 2}$, Guy Van den Mooter ${ }^{\mathrm{a}, *}$ \\ ${ }^{a}$ Drug Delivery and Disposition, KU Leuven, Leuven, Belgium \\ ${ }^{b}$ Biological Formulation Development, UCB Pharma, Braine l'Alleud, Belgium \\ ${ }^{c}$ Non-Clinical Statistics, UCB Pharma, Braine l'Alleud, Belgium \\ ${ }^{d}$ Department of Chemical Engineering, KU Leuven, Leuven, Belgium
}

\begin{abstract}
Electrospraying or electrohydrodynamic atomisation, i.e. the formation of tiny droplets from a jet of conductive liquid under the influence of an electric field, has been gaining in popularity as a particle engineering technique in recent years. In addition to general benefits for particle engineering, e.g. the ability to generate nanometre sized particles with a very narrow size distribution, electrospraying also possesses a number of characteristics, like its applicability at ambient conditions, which could make it especially interesting for formulating therapeutic proteins. However, as fully aqueous solutions of proteins tend to have relatively high electrical conductivities and surface tensions, obtaining a stable Taylor cone-jet mode for these solutions is inherently challenging. This is why in the majority of studies reporting the successful electrospraying of proteins either emulsions, aqueous suspensions or a mixture of water and one or more organic solvents were used instead of fully aqueous solutions. Therefore, an ab initio electrospraying formulation development study was conducted, using only fully aqueous feed solutions containing protein stabilising excipients commonly used in spray- and freeze-drying of therapeutic
\end{abstract}

\footnotetext{
* Corresponding author

Email address: guy.vandenmooter@kuleuven.be (Guy Van den Mooter)

URL: https://gbiomed.kuleuven.be/english/research/50000715/50000716/

(Guy Van den Mooter)

${ }^{1}$ ORCiD: 0000-0003-4245-0782

${ }^{2}$ ORCiD: 0000-0002-9253-9008
}

Preprint submitted to European Journal of Pharmaceutics and BiopharmaceuticsNovember 3, 2019 
proteins. The study included bovine serum albumin (BSA) as a model protein and consisted out of two parts: (1) a one parameter at a time screening study, designed to improve the understanding of how various formulation components influence relevant physicochemical properties and the electrospraying process and (2) two subsequent mixture design of experiments (DoE) studies, designed to aid in the statistical description and prediction of the influence of different protein-excipient combinations on the electrospraying process. Additionally, the influence of physicochemical properties relevant to the electrospraying process, i.e. the volumetric mass density, electrical conductivity, kinematic viscosity and surface tension, was assessed for all feed solutions included in the study.

\section{Keywords:}

Electrospraying, Electrohydrodynamic atomisation, Taylor cone-jet mode, Fully aqueous protein solutions, Protein formulation, Bovine serum albumin, Physicochemical characterisation, Volumetric mass density, Electrical conductivity, Kinematic viscosity, Surface tension, Design of experiments

\section{Introduction}

The first observations regarding the formation of tiny droplets from a jet of conductive liquid under the influence of an electric field, a phenomenon that would later be identified as electrospraying or electrohydrodynamic atomisation, were made over four hundred years ago [1]. Yet, research into the potential of electrospraying as a novel particle engineering technique only started gaining momentum recently, following the Nobel prize being awarded to John B. Fenn for his work on an ionisation method for large biomolecules [2, 3, 4]. Electrospraying owes its general popularity as a particle engineering technique to, i.a., its relatively simple experimental set-up (see Figure 1), its potential to generate nanometre sized particles with very narrow size distributions, its applicability at ambient conditions and the possibility to incorporate it as an atomiser into other drying set-ups like a spray-dryer. However, feed flow rate and thus the throughput of an electrospraying process will be inherently lower than other 
available drying techniques due to the nature of the atomisation process. Recent developments have been made to help remedy the low throughput, such as using multiple nozzles in parallel within a continuous process $[5,6,7,8]$.

Aside from aforementioned general benefits, electrospraying also has a number of characteristics which make it interesting for formulating therapeutic proteins. A main advantage is the applicability of electrospraying at ambient temperatures, which could help minimise the risk of heat induced protein degradation. Additionally, the low feed flow rates, which are generally seen as a disadvantage of the technique, and the principle of atomisation, make that the overall shear stress to which proteins are subjected is relatively low. Finally, the technique is reported to have a very high encapsulation efficiency when using a coaxial set-up. As coaxial electrospraying allows encapsulation without the need for emulsification, it could help limit the interactions between the, often protein denaturing, solvents needed to dissolve the coating material and the protein. This is because an aqueous protein solution could be fed to the inner needle, while the coating solution could be fed to the outer needle, thus limiting contact between both phases $[9,8,4]$.

A major hurdle in the use of electrospraying for protein formulations is that aqueous solutions of proteins tend to have a relatively high electrical conductivity and surface tension, making it generally more challenging to obtain a stable Taylor cone-jet mode (see Figure 3a) compared to the organic solvent-based systems commonly used for electrospraying. The short (cm range) drying distance between the tip of the spray nozzle and the collector, necessary for maintaining an adequate electric field, the high boiling point, and therefore slow evaporation of water, is another challenging factor of using fully aqueous instead of organic solvent based feed solutions $[8,4,10]$. It is because of these hurdles that the majority of the available literature on the electrospraying of biomolecules report processes that either use emulsions [11, 12], an aqueous suspension [13] or a mixture of water and one or more organic solvents [14, 15]. Moerman et al. [16] reported successfully creating protein spots from fully aqueous solutions containing lactate dehydrogenase, glucose-6-phosphate dehydrogenase 
and pyruvate kinase, while López-Rubio and Lagaron [17] succeeded in creating whey protein capsules starting from a fully aqueous solution of this mixture of globular proteins. While the latter publication could serve as a proof of concept, little information is given about relevant physicochemical properties of the feed solution aside from the surface tension and composition.

To the best of our knowledge, no literature exists on electrospraying fully aqueous protein solutions containing commonly used stabilising excipients. Therefore, an ab initio electrospraying formulation development study was conducted, using only fully aqueous feed solutions containing protein stabilising excipients commonly used in spray- and freeze- drying of therapeutic proteins. The study uses bovine serum albumin (BSA) as a model protein. BSA, being a relatively small and stable protein, is a poor reference model for therapeutic proteins when protein stability is in scope. However, assessment of protein stability was beyond the scope of this work, since the focus was on improving the understanding of how various formulation components influence the electrospraying process and relevant physicochemical properties. Therefore, BSA was used as a model protein for this work, as it was deemed to be adequately comparable to therapeutic proteins regarding its impact on the physicochemical properties relevant to the electrospraying process, i.e. the volumetric mass density, electrical conductivity, kinematic viscosity and surface tension. The study is made up of two parts, the first being a one parameter at a time screening study, designed to improve the understanding of how various formulation components influence relevant physicochemical properties and the electrospraying process. Next, a selection of excipients was used in two subsequent mixture design of experiments (DoE) studies, which were designed to aid in the statistical description and prediction of the influence of different protein-excipient combinations on the electrospraying process. 


\section{Materials}

Bovine serum albumin (BSA), sodium phosphate monobasic monohydrate, sodium phosphate dibasic anhydrate, $\mathrm{D}(+)$-trehalose dihydrate, L-histidine, Larginine monohydrochloride, (2-Hydroxypropyl)- $\beta$-cyclodextrin (HP $\beta \mathrm{CD}$ ) and polysorbate 20 were all obtained from Sigma-Aldrich (St. Louis, MO, USA) Hydrochloric acid $(1 \mathrm{~N})$ was purchased from VWR Chemicals (Radnor, PA, USA). Phosphoric acid $85 \%$ (analytical reagent grade) was obtained from Chem-Lab (Zedelgem, Belgium). D(+)-sucrose was obtained from Acros Organic (Geel, Belgium). Hydroxypropylmethylcellulose (HPMC) (type E5) and polyvinylpyrrolidone (PVP) K30 were obtained from Janssen Pharmaceutica (Beerse, Belgium). Sulfobutylether- $\beta$-cyclodextrin sodium salt (Na SBE $\beta C D$ ) was purchased from CyDex Inc. (Lenexa, KS, USA). Polyethylene glycol 300 (PEG 300) was obtained from TCI Chemicals (Portland, OR, USA). PVP K17 and PVP K25 were provided by BASF ChemTrade GmbH (Ludwigshafen, Germany). All electrospraying feed solutions were prepared with ultrapure water (Type $1, \rho \geq 18.2 \mathrm{M} \Omega \mathrm{cm}$ at $298.15 \mathrm{~K}$ ) as the only solvent, i.e. no other solvents or co-solvents were used to prepare the solutions described in this work.

\section{Methods}

\subsection{Electrospraying}

\subsubsection{Preparation of Feed Solutions}

All feed solutions were prepared at ambient temperature (293 $\pm 5 \mathrm{~K})$, using ultrapure water and were magnetically stirred at low speed until a clear solution, free from visible particles, was obtained. Feed solutions were subsequently filtered using either $0.45 \mu \mathrm{m}$ cellulose acetate syringe filters (MachereyNagel, Düren, Germany) or $0.45 \mu \mathrm{m}$ cellulose acetate membrane filters (Sartorius, Göttingen, Germany) under vacuum for the formulations described in Section 4.1 and were not filtered in case of the DoE formulations described in Section 3.7. 


\subsubsection{Electrospraying Process}

Electrospraying was done with a climate-controlled electrospinning machine (EC-CLI) (IME Technologies, Geldrop, the Netherlands) in combination with a PHD 4400 syringe pump (Harvard Apparatus, Holliston, MA, USA) to deliver constant flow rates (see Figure 1). All formulations were electrosprayed using 25 gauge passivated stainless steel dispensing tips (Nordson, Westlake, OH, USA) connected to either $10 \mathrm{~mL}$ or $5 \mathrm{~mL}$ BD PlastiPak syringes with Luer Lock (Becton, Dickinson and Company, Franklin Lakes, NJ, USA) in case of the formulations described in Section 4.1 or Section 3.7, respectively.

Because optimal electrospraying process parameters were expected to differ for each of the screened formulations, process parameters were varied within the ranges shown in Table 1 until the (visibly) most stable cone-jet and driest powder bed were obtained. For the experiments described in Section 4.1, conejet stability or powder bed quality related responses are defined as the values obtained at the optimal process parameter combination for a given formulation.

To visualise the spraying mode at the nozzle during the electrospraying process of the DoE formulations described in Section 3.7, a Fastcam SA2 high speed camera (Photron, Tokyo, Japan) was used, equipped with a K2 DistaMax long distance microscope system and CF-4 objective (Infinity Photo-Optical Company, CO, USA). Recordings were made at a resolution of 2048 x 2048 pixels, a shutter speed of $4 \cdot 10^{-5}$ seconds and a frame rate of 1080 frame per second (fps).

\subsection{Density}

The volumetric mass density is defined as the mass per unit volume. A glass pycnometer with a volume of $10.239 \mathrm{~cm}^{3}$ was used to measure all feed solutions, with each measurement conducted at ambient temperature in a climate controlled room with the temperature set to $293 \mathrm{~K}$. 


\subsection{Surface Tension}

Surface tension was measured using a CAM 200 goniometer and the accompanying CAM 200 software (KSV Instruments, Helsinki, Finland) using the pendant drop method. All solutions were measured at ambient temperature in a climate controlled room with the temperature set to $293 \mathrm{~K}$, using a 25 gauge, passivated stainless steel dispensing tip (Nordson) and surface tension was measured in triplicate for each formulation, i.e. by dispensing three different drops from which 10 pictures were taken at a frame interval of $975 \mathrm{~ms}$, unless explicitly stated otherwise.

For the DoE discussed in Section 3.7.2, an alternative method was used, consisting of collecting 60 frames at 1 fps per droplet and this for five droplets per formulation using an 18 gauge polypropylene dispensing tip (Nordson) in order to avoid wetting the tip's external surface. Subsequently a $t_{0}$ was defined as the point where a droplet is formed and the Young-Laplace equation is valid and a point was determined where a plateau was reached in the surface tension versus time plot, i.e. the point where polysorbate 20 had reached the air-water interface. These points were then compared for all formulations in the DoE and the longest time, i.e. the point where the "slowest" solution reached a plateau and the surface tension values for this time point, relative to $t_{0}$, were entered in the DoE.

\subsection{Viscosity}

The kinematic viscosity of all feed solutions was measured using an iVisc LMV 830 capillary viscometer (Lauda, Köningshofen, Germany) using Ubbelohde capillaries with known volume, diameter and length. Temperature was set at $293 \mathrm{~K}$, using a glass panelled liquid temperature-controlled bath equipped with a Lauda ECO silver immersion thermostat (Lauda).

\subsection{Electrical Conductivity}

An Orion A150+ conductivity meter, equipped with a 013016D conductivity probe with an actual K-value of $0.0923 \mathrm{~cm}^{-1}$ (Thermo Fisher Scientific, 
Walthman, Massachusetts, USA) was used for all electrical conductivity measurements. All solutions were measured at ambient temperature $(293 \pm 5 \mathrm{~K})$ following a two point calibration with $100 \mu \mathrm{S} \mathrm{cm}^{-1}$ and $1413 \mu \mathrm{S} \mathrm{cm}{ }^{-1}$ conductivity standards, which were provided by the manufacturer.

\subsection{Scanning Electron Microscopy}

A JSM-IT300 InTouchScope ${ }^{\circledR}$ scanning electron microscope (SEM) (Jeol, Tokio, Japan), combined with version 1.110 of the IT300 operation software (Jeol), was used to further assess powder bed characteristics. Particle samples were fixed onto a cylindrical mount with double-sided carbon tape and a gold layer was subsequently applied using a Q150R ES Rotary-Pumped Sputter Coater/ Carbon Coater (Quorum Technologies, Lewes, U.K.).

For powder resulting from the DoE formulations described in Section 3.7, an XL30 FEG SEM combined with Genesis 4.61 software (Philips, Amsterdam, The Netherlands) was used, sample preparation was similar to that of the JSMIT300, except for a platinum layer applied instead of the gold layer.

\subsection{Mixture Design of Experiments (DoE)}

In mixture experiments, a factor's value is its proportion in the mixture, which falls between zero and one. Mixture experiments differ from other experimental types in that one cannot vary factors independently of one another. When the proportion of one factor is changed, the proportion of one or more other factors must also change. In our case, we had mixture experiments with either five (Table 3) or three (Table 4) factors, with the sum of these factor proportions being equal to one $(100 \%)$. The final response levels included assessment of both the cone-jet stability and the powder bed quality. An unstable cone-jet should be interpreted as any spraying mode deviating from a Taylor cone-jet mode, i.e. dripping, micro dripping, spindle or multi-jet, but also any droplet elongation or "jerking" of the Taylor cone-jet mode within the time frame observed with the high speed camera or the slow motion recordings 
(> 100 frames at $5 \mathrm{fps}$ ) made herewith. The latter were included in the supporting information of the publication [4]. The powder bed quality was assessed based on whether or not a powder could be obtained and on the number and size of droplet residue visibly present in the powder bed.

\subsubsection{Mixture DoE with Ordinal Responses}

One ordinal response, which combined the observation of both cone-jet and powder bed quality, was of interest and set to be evaluated at five distinct levels, as summarised in Table 5. The suggestions given in the doctoral thesis by Mancenido [18] were used as a starting point to generate the custom design with mixture factors displayed in Table 3 using the JMP 11.0 software package (SAS Institute, Cary, North Carolina, USA). BSA, glycerol, trehalose, HP $\beta$ CD and PVP K25 concentrations were selected as the five factors for the subsequent mixture DoE, which was based on a custom design generated in JMP 11.0. The resulting DoE is summarized in Table 3 . Only main effects can be estimated with the generated design. DoE encoding used in Table 3 represents the fraction of a $40 \% \mathrm{~m} / \mathrm{V}$ (40 g of solute per $100 \mathrm{~mL}$ of aqueous solution) excipient content, not including polysorbate $20(1 \% \mathrm{~m} / \mathrm{V})$, that each formulation factor assumes. As will be shown in Section 4.2, two binary responses were used instead of an ordinal response in the final data analysis. A detailed description of the statistical methodology is included in the supporting information.

\subsubsection{Mixture DoE with Three Factors for Binary Responses}

As for the method described in Section 3.7.1, two binary responses are registered for this design: cone-jet stability and powder bed rating. The levels of the responses are described in Table 6. BSA, HP $\beta$ CD and PVP K25 concentrations were selected as the three factors for the subsequent mixture DoE, which was based on a simplex lattice algorithm generated in JMP 11.0. The resulting DoE is summarised in Table 4 where DoE encoding represents the fraction of a $20 \% \mathrm{~m} / \mathrm{V}$ excipient content, not including trehalose $(20 \% \mathrm{~m} / \mathrm{V})$ and polysorbate $20(1 \% \mathrm{~m} / \mathrm{V})$, that each formulation factor assumes. Similarly to the 
method described in Section 3.7.1, logistic regression was applied and model estimation was done using the maximum likelihood. A detailed description of the statistical methodology is included in the supporting information.

\section{Results and Discussion}

\subsection{Electrospraying Feasibility Screening}

A large set of excipients commonly used for stabilising spray- or freeze-dried protein formulations, either in solid state or in liquid state during drying, was selected in an ab initio formulation development for fully aqueous feed solutions for electrospraying. The relevant physicochemical characteristics of the feed solutions, i.e. volumetric mass density, electrical conductivity, kinematic viscosity and surface tension, were determined where possible to increase understanding of the influence of different formulation components. In this regard, it is worth pointing out that the $\mathrm{pH}$ of the different solutions was not determined during the experiments, even though the $\mathrm{pH}$ of a solution determines the total amount of charged groups a protein carries and thus influences the protein's molecular organization and stability in solution. But, as mentioned in the introduction (see Section 1), assessment of the protein stability was beyond the scope of the present work. The main responses used for the electrospraying feasibility screening experiments were the quality of the powder bed, e.g. whether or not solid particles could be obtained, if there were any droplets deposited on the collector during the process, and visual assessment of the cone-jet stability. Overall, the main focus of this part of the study was on whether or not solid particles could be collected, since directly assessing the cone-jet stability was not always feasible with the set-up used for these experiments.

Results of the broad electrospraying feasibility screening study (see supporting information) corroborated findings of Kriegel et al. [19] and Pérez-Masiá et al. [20], which stated that decreasing the surface tension results in a lower electrical field strength being required for jet initiation, thus improving cone-jet feasibility. The findings also confirm the theoretical surface tension "cut-off" 
value of circa $50 \mathrm{mN} \mathrm{m}^{-1}$ [21]. Nevertheless, one of the biggest challenges of electrospraying aqueous protein solutions lies in their (relatively) high electrical conductivity, which could be countered by reducing the overall surface tension of the liquid, as surface tension and conductivity have counteracting effects regarding the electrospraying process. Furthermore, the electrospraying experiments summarised in the supporting information revealed that an increase in PVP K25 concentration positively affected the formulation's electrospraying feasibility, i.e. a visibly more stable cone-jet was observed with less droplets deposited in the powder bed. Yet, little difference could observed between different types of PVP concerning the electrospraying process characteristics. Improvement of the powder bed quality with concentration of the non-ionisable PVP K25 was reminiscent of the results observed for increasing BSA concentrations. However, contrary to PVP, electrical conductivity increased with increasing BSA concentrations (see supporting information). These findings emphasized the importance of the total solids content present in the feed solution as an additional requirement for ensuring adequate drying efficiency during electrospraying.

With regard to process parameters, it was found that the best results were generally obtained when a feed flow rate of $0.2 \mathrm{~mL}$ per hour and a tip to collector (TTC) distance between 6 and $12 \mathrm{~cm}$ were applied. Larger TTC distances resulted in an increased amount of droplets on the collector, as the electric field strength required for jet initiation became too weak at these distances. Generally, a voltage between $19 \mathrm{kV}$ and $25 \mathrm{kV}$ was needed to obtain solid particles on the collector. However, as illustrated by the SEM images obtained for different fractions of formulation K25_5 (see supporting information), the powders resulting from the one parameter at a time screening study still displayed substantial heterogeneity.

\subsection{Mixture DoE with Ordinal Responses}

A mixture DoE was chosen to allow for a more in depth assessment of how the different formulation components influence the process and the resulting powder quality. Following the execution of the DoE, it was found to be hard to 
divide the defined response, which combined cone-jet stability and powder bed quality observations, into five categories as originally intended (see Table 5). Therefore, as differences in cone-jet stability and powder bed quality were less pronounced than expected, two separate responses were recorded and, for each response, two factor levels were defined (see Table 6). Given this encoding revision of the responses, a logistic regression was applied during data analysis.

To further improve the resolution of our process quality assessment, a high speed camera was used to assess cone-jet stability instead of the previously used visual observation method. Table 7 summarises the physicochemical characteristics and response values obtained for the DoE given in Table 3. Values obtained for the cone-jet stability and powder bed responses were identical for all runs, implying that the estimations for both models are identical as well. The response values were subsequently processed according to the method described in Section 3.7.1, but among the fifteen runs performed for this DoE, only five gave a level 2 response. This resulted in the obtained estimators (Table 8) being "unstable", i.e. they could not be considered as reliable due to the very large standard error of the obtained parameter estimates.

Figure 3 illustrates how a difference in cone-jet stability resulted in a different powder bed morphology, as formulation M2 yielded nanometre-sized and highly monodisperse particles, resemblant of the carbamazepine nanoparticles obtained by Wang et al. [22], while the unstable cone-jet resulting from formulation M14 gave particles more similar to those obtained with a spray-drying process. Additionally, slow motion recordings ( $>100$ frames at $5 \mathrm{fps}$ ) of the cone-jet modes made with the high speed camera are included in the supporting information of the publication. It should be noted that the overall integrity of powder/particle morphology was relatively low. This resulted in a large part of the formulations being converted to agglomerates or homogeneous layers on the aluminium foil (used for collection) awaiting SEM analysis, despite being stored in a desiccator at $278 \mathrm{~K}$. The low storage integrity of these powders is believed to be caused by a combination of the very small particle sizes obtained with the process and high residual water content in the powder bed, both in- 
side and around the particles. In order to confirm this hypothesis, additional characterisation of the powders, e.g. Karl Fischer titration, thermogravimetric analysis,... will have to be performed, which was beyond the scope of the present study due to sample quantity restrictions. To resolve this issue, a different set-up could be used to increase the drying distance without having to sacrifice electric field strength. An example could be the electrospraying set-up used by Ciach [5], who used a nozzle-ring set-up inside a glass chamber under air flow, a process very much resembling spray-drying, where electrospraying functions as the atomiser. Although promising, feed flow rates for this process will still be much lower than those obtainable with a standard spray-drying setup, and the added effect of the air flow on the electrospraying process should not be underestimated. Alternatively, the electrospraying process could be performed at elevated temperatures and decreased humidity. Even though this would increase the rate of water evaporation, temperature changes could heavily impact the physicochemical properties of the feed solution, e.g. viscosity, electrical conductivity, ..., making process optimisation and stringent temperature control necessary for such processes. The use of higher temperatures would also nullify the advantage of working at ambient conditions and could introduce additional solid state stability concerns. Finally, a more aggressive post-drying step than storage in a desiccator could be added to the method, like freeze-drying the final product, but this would once again nullify some of the advantages that electrospraying has compared to more traditional drying processes like spray- and freeze-drying. Regardless of which of these approaches is selected, a thorough understanding of the impact of formulation and process parameters on the electrospraying process will be indispensable.

In addition to assessing the influence of formulation factors on the conejet stability and powder bed responses, the influence of the physicochemical (uncontrolled) factors shown in Table 7 was evaluated by both plotting the responses versus physicochemical factors and by including them as factors in regression models. However, neither method was able to show a correlation between, or a significant influence on, the different responses for any of the 
included physicochemical parameters.

Despite not yielding any statistically significant model, the collected data (see Table 7) did show that glycerol seemed to impact the responses, more specifically, that the absence of glycerol in the mixture correlated to a response equal to 2. Additionally, it can be observed that all responses which equal 2 contain $20 \% \mathrm{~m} / \mathrm{V}$ trehalose. Therefore, glycerol was eliminated from formulations for the mixture DoE described in Section 3.7.2, while $20 \% \mathrm{~m} / \mathrm{V}$ trehalose was added to all formulations, thus leaving only BSA, HP $\beta$ CD and PVP K25 as factors of the resulting mixture DoE with three factors for binary responses (see Section 3.7.2) of which the results are given in Section 4.3.

\subsection{Mixture DoE with Three Factors for Binary Responses}

Following the analysis of the mixture design defined in Section 3.7.1, three formulation factors were selected to be further analysed: $\mathrm{BSA}, \mathrm{HP} \beta \mathrm{CD}$ and PVP K25, according to the mixture DoE, based on simplex lattice algorithm generated in JMP 11.0. The resulting DoE is summarised in Table 4 where DoE encoding represents the fraction of a $20 \% \mathrm{~m} / \mathrm{V}$ excipient content, not including trehalose $(20 \% \mathrm{~m} / \mathrm{V})$ and polysorbate $20(1 \% \mathrm{~m} / \mathrm{V})$, that each formulation factor assumes.

Table 9 summarises the results obtained for the DoE given in Table 4. Contrary to Section 4.2, there are discrepancies between the "Cone-jet stability rating" and "Powder bed rating" response values, which is likely due to the resolution of powder bed observation being too low to distinguish between subtle differences in powder bed quality, resulting from slightly different cone-jet stability modes observed with the high speed camera. This was within expectations as the formulations included in the DoE in scope could be considered as being more optimised for electrospraying than those discussed in Section 4.2, thus making the difference between different formulations less pronounced. Following data acquisition, response values were processed according to the method described in Section 3.7.2. Additionally, slow motion recordings ( $>100$ frames at $5 \mathrm{fps}$ ) of the cone-jet modes made with the high speed camera are included 
in the supporting information of the publication. Representative SEM images could not be taken for any of the formulations due to powder/particle integrity issues, as was discussed in Section 4.2 and illustrated by the SEM images shown in Figure 6.

Estimator values for the cone-jet stability response are given in Table 10, initial analysis of the DoE showed BSA to be borderline significant with a $p$ value (Likelihood Ratio (LR) test) of 0.0519 (see Table 10). Figure 4 represents the JMP prediction profiler and associated desirability functions output which were used to determine the factor value combination, which, according to the model, would have the highest probability of getting a response equal to 2 . The optimum mixture combination that should theoretically maximise the odds was determined to be $30.7 \%$ (DoE encoding) or $6.2 \% \mathrm{~m} / \mathrm{V}$ BSA, $69.3 \%$ (DoE encoding) or $13.8 \%$ m/V PVP K25 and $0 \%$ HP $\beta$ CD (see Figure 4).

The estimated optimal feed solution was then prepared in triplicate (L22, L23 and L24), characterised and electrosprayed. All three iterations gave a value of 2 for both responses. Response values and physicochemical characteristics can be found in Table 9. The results of these three confirmatory runs were subsequently added to the original DoE and results were reanalysed with these values included. Estimator and $p$-values of this expanded DoE are summarised in Table 11 for the cone-jet stability response, confirming the statistical significance of the BSA factor effect, which now has a $p$-value (LR test) of 0.0319 (see Table 11). The JMP prediction profiler and the associated desirability function are presented in Figure 5 and reconfirm the previously found optimum values for the cone-jet stability response at $31.4 \%$ (DoE encoding) or $6.3 \% \mathrm{~m} / \mathrm{V}$ BSA, $68.5 \%$ (DoE encoding) or $13.7 \% \mathrm{~m} / \mathrm{V}$ PVP K25 and $0 \% \mathrm{HP} \beta \mathrm{CD}$.

Data processing of the powder bed response was conducted in the same manner as for the cone-jet stability response, but estimators for the powder bed response (see supporting information) were "unstable" due to the very large standard error of the obtained parameter estimates, making their values unreliable. $p$-Values resulting from the Wald and LR test are thus included for information purposes only. The three confirmatory runs all gave a level 2 powder 
bed response, confirming that a good powder bed was obtained at the theoretical mixture optimum, but the addition of the confirmatory runs to the dataset did not result in a reliable model as the standard error remained large (see supporting information).

Assessment of the physicochemical data (see Table 9) was performed in the same way as for the first DoE (see Section 4.2) for both responses, i.e. by both plotting responses versus physicochemical factors and by including them as factors in regression models. However, neither method was able to show a correlation between or a significant influence on the different responses for any of the physicochemical parameters included in the study.

Overall, the presented results provide evidence that electrospraying of fully aqueous BSA solutions is feasible and that, using the correct statistical approach, the atomisation process can be predicted and optimised for formulations containing BSA. Even so, the used statistical approach did not allow for prediction of powder bed quality or estimation of the impact of, or interaction between, different physicochemical characteristics of the feed solution. The latter was likely due to the fact that physicochemical factors had to be analysed as "uncontrolled factors" in the DoE, rather than formulating feed solutions possessing a range of different physiochemical properties. Visual observation of the powder bed quality was probably insufficiently discriminatory between good and bad processes, but was deemed the most feasible for this study, due to the high number of experiments and low process throughput. Hence, it is suggested that future work should include a more complete set of characterisation methods like particle size, morphology, water content, ... to increase the overall response resolution. Protein stability of the formulations was not taken into account for the present work, inclusion of these parameters in future work, with a more therapeutically relevant model protein, will likely introduce a number of additional constraints and requirements to ensure production of a high quality protein powder [23]. 


\section{Conclusions}

The present body of work has confirmed the feasibility of electrospraying fully aqueous solutions containing BSA in order to obtain a powder, albeit one with limited storage integrity. Extrapolation of the general conclusions of this study to other, more therapeutically relevant, proteins seems permissible, provided that there is sufficient similarity with BSA for relevant physicochemical characteristics. A caveat here is that protein instability and aggregation could be disruptive factors. A design of experiments approach was successfully used to screen and optimise the electrospraying performance of a formulation based on the Taylor cone-jet stability. No valid statistical model could be obtained for the powder bed quality, but experiments did suggest the powder bed quality being dependent on the amount of dissolved solids present in the feed solution. The overall (storage) integrity of the obtained powders was low, presumably as a consequence of a high residual water content still being present after drying, a hypothesis that still needs to be confirmed, which could be resolved by further optimising the process parameters or making alterations to the used set-up. Finally, an extensive dataset containing physicochemical properties relevant to electrospraying was gathered for a large number of excipients commonly used to stabilise proteins in spray- or freeze-drying, which can be used as a reference work for future studies.

\section{Acknowledgements}

The authors would like to thank UCB Pharma for providing the equipment, materials and funding to make this study possible.

\section{References}

\section{References}

[1] W. Gilbert of Colchester, De Magnete, Magneticisque Corporibus, et de Magno Magnete Tellure, 1st Edition, Peter Short, London, UK, 1600. 
[2] M. Yamashita, J. B. Fenn, Electrospray Ion Source. Another Variation on the Free-Jet Theme, The Journal of Physical Chemistry 88 (20) (1984) 4451-4459. arXiv:https://doi.org/10.1021/j150664a002, doi: $10.1021 / \mathrm{j} 150664 \mathrm{a} 002$.

[3] J. B. Fenn, M. Mann, C. K. Meng, S. F. Wong, C. M. Whitehouse, Electrospray Ionization for Mass Spectrometry of Large Biomolecules, Science 246 (4926) (1989) 64-71. doi:10.1126/science.2675315.

[4] A. Smeets, C. Clasen, G. Van den Mooter, Electrospraying of Polymer Solutions: Study of Formulation and Process Parameters, European Journal of Pharmaceutics and Biopharmaceutics 119 (2017) 114-124. doi: 10.1016/j.ejpb.2017.06.010.

[5] T. Ciach, Microencapsulation of Drugs by Electro-Hydro-Dynamic Atomization, International Journal of Pharmaceutics 324 (1) (2006) 51-55. doi:10.1016/j.ijpharm.2006.06.035.

[6] N. Bock, T. Dargaville, M. Woodruff, Electrospraying of Polymers with Therapeutic Molecules: State of the Art, Progress in Polymer Science 37 (11) (2012) 1510-1551. doi:10.1016/j.progpolymsci.2012.03.002.

[7] J. Xie, J. Jiang, P. Davoodi, M. Srinivasan, C.-H. Wang, Electrohydrodynamic Atomization: A Two-Decade Effort to Produce and Process Micro/Nanoparticulate Materials, Chemical Engineering Science 125 (2015) 3257. doi:10.1016/j.ces.2014.08.061.

[8] D. N. Nguyen, C. Clasen, G. Van den Mooter, Pharmaceutical Applications of Electrospraying, Journal of Pharmaceutical Sciences 105 (9) (2016) 26012620. doi:10.1016/j.xphs.2016.04.024.

[9] L. Zhang, J. Huang, T. Si, R. X. Xu, Coaxial Electrospray of Microparticles and Nanoparticles for Biomedical Applications, Expert review of medical devices 9 (6) (2012) 595-612. doi:10.1586/erd.12.58. 
[10] C. G. Drosou, M. K. Krokida, C. G. Biliaderis, Encapsulation of Bioactive Compounds Through Electrospinning/Electrospraying and Spray Drying: A Comparative Assessment of Food-Related Applications, Drying Technology 35 (2) (2017) 139-162. arXiv:http://dx.doi.org/10.1080/ 07373937.2016.1162797, doi:10.1080/07373937.2016.1162797.

[11] T. Suksamran, P. Opanasopit, T. Rojanarata, T. Ngawhirunpat, U. Ruktanonchai, P. Supaphol, Biodegradable Alginate Microparticles Developed by Electrohydrodynamic Spraying Techniques for Oral Delivery of Protein, Journal of Microencapsulation 26 (7) (2009) 563-570. doi:10.3109/ 02652040802500622.

[12] T. Suksamran, T. Ngawhirunpat, T. Rojanarata, W. Sajomsang, T. Pitaksuteepong, P. Opanasopit, Methylated N-(4-N,N-Dimethylaminocinnamyl) Chitosan-Coated Electrospray OVA-Loaded Microparticles for Oral Vaccination, International Journal of Pharmaceutics 448 (1) (2013) 19-27. doi:10.1016/j.ijpharm.2013.03.015.

[13] S. Saallah, M. N. Naim, M. N. Mokhtar, N. F. A. Bakar, M. Gen, I. W. Lenggoro, Transformation of Cyclodextrin Glucanotransferase (CGTase) from Aqueous Suspension to Fine Solid Particles Via Electrospraying, Enzyme and Microbial Technology 64-65 (2014) 52-59. doi:10.1016/j. enzmictec.2014.06.002.

[14] A. Gomez, D. Bingham, L. de Juan, K. Tang, Production of Protein Nanoparticles by Electrospray Drying, Journal of Aerosol Science 29 (5) (1998) 561-574. doi:10.1016/S0021-8502 (97)10031-3.

[15] R. Pareta, A. Brindley, M. J. Edirisinghe, S. N. Jayasinghe, Z. B. Luklinska, Electrohydrodynamic Atomization of Protein (Bovine Serum Albumin), Journal of Materials Science: Materials in Medicine 16 (10) (2005) 919-925. doi:10.1007/s10856-005-4426-z.

[16] R. Moerman, J. Frank, J. C. M. Marijnissen, T. G. M. Schalkhammer, G. W. K. van Dedem, Miniaturized Electrospraying as a Technique for 
the Production of Microarrays of Reproducible Micrometer-Sized Protein Spots, Analytical Chemistry 73 (10) (2001) 2183-2189. doi:10.1021/ ac001041g.

[17] A. López-Rubio, J. M. Lagaron, Whey Protein Capsules Obtained Through Electrospraying for the Encapsulation of Bioactives, Innovative Food Science and Emerging Technologies 13 (2012) 200-206. doi:10.1016/j. ifset.2011.10.012.

[18] M. V. Mancenido, Categorical Responses in Mixture Experiments, Doctoral Dissertation, Arizona State University (2016).

[19] C. Kriegel, K. M. Kit, D. J. McClements, J. Weiss, Influence of Surfactant Type and Concentration on Electrospinning of Chitosan-Poly(Ethylene Oxide) Blend Nanofibers, Food Biophysics 4 (3) (2009) 213-228. doi: $10.1007 / \mathrm{s} 11483-009-9119-6$.

[20] R. Pérez-Masiá, J. M. Lagaron, A. López-Rubio, Surfactant-Aided Electrospraying of Low Molecular Weight Carbohydrate Polymers from Aqueous Solutions, Carbohydrate Polymers 101 (2014) 249-255. doi:10.1016/j. carbpol.2013.09.032.

[21] R. Hartman, D. Brunner, D. Camelot, J. Marijnissen, B. Scarlett, Electrohydrodynamic Atomization in the Cone-Jet-Mode Physical Modeling of the Liquid Cone and Jet., Journal of Aerosol Science 30 (7) (1999) 823-849. doi:10.1016/S0021-8502(99)00033-6.

[22] M. Wang, G. C. Rutledge, A. S. Myerson, B. L. Trout, Production and Characterization of Carbamazepine Nanocrystals by Electrospraying for Continuous Pharmaceutical Manufacturing, Journal of Pharmaceutical Sciences 101 (3) (2012) 1178-1188. doi:10.1002/jps. 23024.

[23] C. J. Roberts, Therapeutic Protein Aggregation: Mechanisms, Design, and Control, Trends in Biotechnology 32 (7) (2014) 372-380. doi:10.1016/j. tibtech.2014.05.005. 
Table 1: Range of electrospraying process parameters scanned in search for the experiments.

Parameter values denoted with an * were not varied, but fixed at the value shown in the table.

\begin{tabular}{lll}
\hline Parameter & Screening study & Mixture DoE studies \\
\hline Voltage & $0-25 \mathrm{kV}$ & $19-25 \mathrm{kV}$ \\
Flow rate & $0.1-3 \mathrm{~mL} /$ hour & $0.1 \mathrm{~mL} /$ hour * \\
Tip to collector distance & $6-15 \mathrm{~cm}$ & $9 \mathrm{~cm}^{*}$ \\
Temperature & $298 \pm 2 \mathrm{~K}^{*}$ & $303 \pm 2 \mathrm{~K}^{*}$ \\
Relative humidity & $35 \pm 5 \%$ (fixed) & $20 \pm 5 \%^{*}$ \\
\hline
\end{tabular}

Table 2: Aqueous formulations containing different concentrations and types of PVP in the presence of BSA.

\begin{tabular}{|c|c|c|c|c|}
\hline \multirow[t]{2}{*}{ Formulation } & \multicolumn{2}{|c|}{ PVP } & \multirow{2}{*}{$\begin{array}{c}\mathrm{BSA} \\
\% \mathrm{~m} / \mathrm{V}\end{array}$} & \multirow{2}{*}{$\begin{array}{c}\text { Polysorbate } 20 \\
\% \mathrm{~m} / \mathrm{V}\end{array}$} \\
\hline & Type & $\% \mathrm{~m} / \mathrm{V}$ & & \\
\hline $\mathrm{K} 25 \_2$ & $\mathrm{~K} 25$ & 2.0 & 10 & 1.105 \\
\hline K25_3 & $\mathrm{K} 25$ & 3.0 & 10 & 1.105 \\
\hline K25_5 & $\mathrm{K} 25$ & 5.0 & 10 & 1.105 \\
\hline K17_5 & K17 & 5.0 & 10 & 1.105 \\
\hline K30_5 & K30 & 5.0 & 10 & 1.105 \\
\hline
\end{tabular}


Table 3: Mixture DoE with replicate runs (factors in DoE encoding ).

\begin{tabular}{lccccc}
\hline Formulation & BSA & Glycerol & Trehalose & HP $\beta$ CD & PVP K25 \\
\hline M1 & 0.025 & 0.05 & 0.5 & 0.175 & 0.25 \\
M2 & 0.025 & 0 & 0.5 & 0.475 & 0 \\
M3 & 0.25 & 0 & 0 & 0.5 & 0.25 \\
M4 & 0.25 & 0 & 0.5 & 0 & 0.25 \\
M5 & 0.25 & 0.05 & 0.2 & 0.5 & 0 \\
M6 & 0.25 & 0.05 & 0.45 & 0 & 0.25 \\
M7 & 0.025 & 0.05 & 0.175 & 0.5 & 0.25 \\
M8 & 0.25 & 0 & 0 & 0.5 & 0.25 \\
M9 & 0.25 & 0 & 0.5 & 0 & 0.25 \\
M10 & 0.025 & 0 & 0.5 & 0.475 & 0 \\
M11 & 0.25 & 0.05 & 0.5 & 0.2 & 0 \\
M12 & 0.25 & 0.05 & 0.2 & 0.5 & 0 \\
M13 & 0.025 & 0 & 0.5 & 0.475 & 0 \\
M14 & 0.25 & 0 & 0 & 0.5 & 0.25 \\
M15 & 0.025 & 0.05 & 0.175 & 0.5 & 0.25 \\
\hline
\end{tabular}

${ }^{\mathrm{a}}$ DoE encoding represents the fraction of a $40 \% \mathrm{~m} / \mathrm{V}$ excipient content, not including polysorbate $20(1 \% \mathrm{~m} / \mathrm{V})$, that each formulation factor assumes. 
Table 4: Mixture DoE following a simplex lattice (factors in DoE encoding ).

\begin{tabular}{lccc}
\hline Formulation & BSA & HP $\beta$ CD & PVP K25 \\
\hline L1 & 0.2 & 0.4 & 0.4 \\
L2 & 0.6 & 0 & 0.4 \\
L3 & 0 & 0.2 & 0.8 \\
L4 & 0 & 0 & 1 \\
L5 & 0.2 & 0.6 & 0.2 \\
L6 & 0.6 & 0.2 & 0.2 \\
L7 & 0.2 & 0.8 & 0 \\
L8 & 0 & 0.4 & 0.6 \\
L9 & 0.4 & 0.6 & 0 \\
L10 & 0 & 0.6 & 0.4 \\
L11 & 0.2 & 0.2 & 0.6 \\
L12 & 0.4 & 0.2 & 0.4 \\
L13 & 0.8 & 0.2 & 0 \\
L14 & 0.6 & 0.4 & 0 \\
L15 & 0.2 & 0 & 0.8 \\
L16 & 0 & 0.8 & 0.2 \\
L17 & 0.8 & 0 & 0.2 \\
L18 & 0.4 & 0.4 & 0.2 \\
L19 & 0 & 1 & 0 \\
L20 & 0.4 & 0 & 0.6 \\
L21 & 1 & 0 & 0 \\
\hline & & & \\
\hline
\end{tabular}

${ }^{a}$ DoE encoding represents the fraction of a $20 \% \mathrm{~m} / \mathrm{V}$ excipient content, not including trehalose $(20 \% \mathrm{~m} / \mathrm{V})$ and polysorbate 20 $(1 \% \mathrm{~m} / \mathrm{V})$, that each formulation factor assumes. 
Table 5: Original response level encoding for the mixture DoE summarised in Table 3.

\begin{tabular}{cl} 
Level & Observation of cone-jet and powder bed quality \\
\hline 1 & Thick water jet, no mist \\
2 & Sprayed mist, no solid \\
3 & Sprayed mist, solid with thick droplets \\
4 & Sprayed mist, solid with fine droplets in powder bed \\
5 & Sprayed mist, dry powder without visible droplets \\
\hline
\end{tabular}

Table 6: Final response level encoding for the mixture DoE summarised in Table 3 and Table 4.

\begin{tabular}{cll}
\hline Level & Cone-jet stability rating & Powder bed rating \\
\hline 1 & Unstable within the observed time & Many and/or large droplets \\
& frame & present \\
2 & Stable within the observed time & Visually dry \\
& frame & \\
\hline
\end{tabular}


Table 7: Results for the mixture DoE (see Table 3) with replicate runs and the measured physicochemical properties associated with each formulation.

\begin{tabular}{lcccccccccc}
\hline & \multicolumn{2}{c}{\begin{tabular}{c} 
Density \\
\multicolumn{2}{c}{$\mathrm{g} \mathrm{cm}^{-3}$}
\end{tabular}} & $\begin{array}{c}\text { Surface tension } \\
\mathrm{mN} \mathrm{m}^{-1}\end{array}$ & $\begin{array}{c}\text { Kin. viscosity } \\
\mathrm{mm}^{2} \mathrm{~s}^{-1}\end{array}$ & $\begin{array}{c}\text { El. conductivity } \\
\mu \mathrm{Scm}^{-1}\end{array}$ & $\begin{array}{c}\text { Cone-jet } \\
\text { stability }\end{array}$ & $\begin{array}{c}\text { Powder } \\
\text { bed }\end{array}$ \\
& $n=3$ & $\pm \mathrm{SD}$ & $n=3$ & $\pm \mathrm{SD}$ & $n=3$ & $\pm \mathrm{SD}$ & $n=3$ & $\pm \mathrm{SD}$ & rating & rating \\
\hline M1 & 1.12637 & 0.00076 & 31.412 & 0.161 & 18.06 & 0.34 & 154.1 & 1.1 & 1 & 1 \\
M2 & 1.13513 & 0.00064 & 34.385 & 0.339 & 5.55 & 0.01 & 93.8 & 0.4 & 2 & 2 \\
M3 & 1.10676 & 0.00085 & 37.601 & 0.204 & 33.96 & 0.24 & 471.7 & 1.2 & 1 & 1 \\
M4 & 1.12339 & 0.00091 & 34.806 & 0.371 & 22.97 & 0.03 & 431.3 & 2.5 & 2 & 2 \\
M5 & 1.11992 & 0.00140 & 35.843 & 0.224 & 8.35 & 0.02 & 427.0 & 1.0 & 1 & 1 \\
M6 & 1.12049 & 0.00051 & 34.128 & 0.698 & 23.52 & 0.15 & 436.3 & 1.5 & 1 & 1 \\
M7 & 1.11427 & 0.00050 & 35.444 & 0.357 & 20.25 & 0.01 & 185.4 & 0.2 & 1 & 1 \\
M8 & 1.10466 & 0.00056 & 37.192 & 0.603 & 31.78 & 0.20 & 477.0 & 3.5 & 1 & 1 \\
M9 & 1.12321 & 0.00050 & 34.442 & 0.899 & 23.26 & 0.32 & 432.7 & 1.2 & 2 & 2 \\
M10 & 1.13660 & 0.00013 & 34.826 & 0.211 & 5.73 & 0.02 & 93.6 & 0.5 & 2 & 2 \\
M11 & 1.13144 & 0.00020 & 31.709 & 0.432 & 7.14 & 0.00 & 399.3 & 0.6 & 1 & 1 \\
M12 & 1.11931 & 0.00036 & 36.005 & 0.390 & 8.11 & 0.00 & 433.3 & 0.6 & 1 & 1 \\
M13 & 1.13584 & 0.00099 & 34.813 & 0.450 & 5.78 & 0.04 & 94.2 & 1.1 & 2 & 2 \\
M14 & 1.10356 & 0.00012 & 37.434 & 0.211 & 30.03 & 0.14 & 474.7 & 1.5 & 1 & 1 \\
M15 & 1.11408 & 0.00070 & 35.913 & 0.044 & 20.82 & 0.26 & 185.1 & 0.5 & 1 & 1 \\
\hline
\end{tabular}


Table 8: Parameter estimates, Wald and Likelihood Ratio (LR) test for the mixture DoE with replicate runs.

\begin{tabular}{lccccc}
\hline \multirow{2}{*}{ Term } & \multirow{2}{*}{ Estimate } & \multirow{2}{*}{ Standard error } & \multicolumn{2}{c}{$p$-value } & \multirow{2}{*}{ Remark } \\
& & & Wald test & LR test & \\
\hline BSA & 17.424016 & 8966.3688 & 0.9984 & 0.9978 & Unstable \\
Glycerol & 660.165053 & 50255.443 & 0.9895 & $0.0012^{*}$ & Unstable \\
Trehalose & -49.607099 & 4271.6647 & 0.9907 & 0.9738 & Unstable \\
HP $\beta$ CD & 15.4189488 & 4391.8467 & 0.9972 & 0.9969 & Unstable \\
PVP K25 & 17.1407206 & 8264.7876 & 0.9983 & 0.9976 & Unstable \\
\hline
\end{tabular}


Table 9: Results for the mixture DoE following a simplex lattice (see Table 4) with replicate runs and the measured physicochemical properties associated with each formulation including 3 additional confirmation points (L22, L23 and L24).

\begin{tabular}{|c|c|c|c|c|c|c|c|c|c|c|}
\hline & \multicolumn{2}{|c|}{$\begin{array}{l}\text { Density } \\
\mathrm{g} \mathrm{cm}^{-3}\end{array}$} & \multicolumn{2}{|c|}{$\begin{array}{l}\text { Surface tension } \\
\qquad \mathrm{mN} \mathrm{m}^{-1}\end{array}$} & \multicolumn{2}{|c|}{$\begin{array}{l}\text { Kin. viscosity } \\
\mathrm{mm}^{2} \mathrm{~s}^{-1}\end{array}$} & \multicolumn{2}{|c|}{$\begin{array}{l}\text { El. conductivity } \\
\mu \mathrm{S} \mathrm{cm}^{-1}\end{array}$} & \multirow{2}{*}{$\begin{array}{c}\text { Cone-jet } \\
\text { stability } \\
\text { rating }\end{array}$} & \multirow{2}{*}{$\begin{array}{c}\text { Powder } \\
\text { bed } \\
\text { rating }\end{array}$} \\
\hline & $n=3$ & $\pm \mathrm{SD}$ & $n=3$ & $\pm \mathrm{SD}$ & $n=3$ & $\pm \mathrm{SD}$ & $n=3$ & $\pm \mathrm{SD}$ & & \\
\hline L1 & 1.12793 & 0.00046 & 32.826 & 0.069 & 16.26 & 0.16 & 225.0 & 1.7 & 2 & 2 \\
\hline L2 & 1.12601 & 0.00079 & 35.044 & 0.122 & 20.45 & 0.04 & 472.3 & 0.6 & 2 & 2 \\
\hline L3 & 1.12222 & 0.00044 & 31.778 & 0.048 & 30.96 & 0.49 & 136.3 & 0.0 & 1 & 2 \\
\hline L4 & 1.11814 & 0.00036 & 32.191 & 0.349 & 38.97 & 0.59 & 145.8 & 0.9 & 2 & 2 \\
\hline L5 & 1.13124 & 0.00008 & 33.999 & 0.061 & 9.98 & 0.12 & 202.0 & 2.0 & 2 & 1 \\
\hline L6 & 1.12845 & 0.00046 & 32.763 & 0.047 & 13.11 & 0.06 & 464.0 & 2.6 & 2 & 1 \\
\hline L7 & 1.13487 & 0.00030 & 33.439 & 0.049 & 6.04 & 0.00 & 183.2 & 1.1 & 2 & 2 \\
\hline L8 & 1.12612 & 0.00068 & 32.585 & 0.151 & 20.20 & 0.21 & 120.0 & 1.1 & 1 & 2 \\
\hline L9 & 1.13402 & 0.00086 & 33.891 & 0.103 & 6.72 & 0.01 & 313.7 & 2.3 & 1 & 1 \\
\hline L10 & 1.12950 & 0.00032 & 33.650 & 0.059 & 14.59 & 0.02 & 104.4 & 1.1 & 2 & 2 \\
\hline L11 & 1.12614 & 0.00018 & 31.760 & 0.302 & 24.25 & 0.06 & 238.3 & 1.2 & 1 & 2 \\
\hline L12 & 1.12772 & 0.00028 & 32.402 & 0.051 & 18.29 & 0.04 & 338.3 & 1.2 & 2 & 2 \\
\hline L13 & 1.13150 & 0.00060 & 33.113 & 0.095 & 9.38 & 0.03 & 603.3 & 3.5 & 1 & 1 \\
\hline L14 & 1.13434 & 0.00029 & 32.014 & 0.095 & 7.99 & 0.00 & 452.3 & 1.2 & 1 & 1 \\
\hline L15 & 1.12085 & 0.00064 & 34.232 & 0.076 & 31.79 & 0.27 & 263.0 & 1.0 & 2 & 2 \\
\hline L16 & 1.13358 & 0.00031 & 33.554 & 0.061 & 9.41 & 0.17 & 85.4 & 0.2 & 1 & 1 \\
\hline L17 & 1.13339 & 0.00056 & 33.915 & 0.207 & 17.68 & 0.07 & 591.0 & 6.2 & 1 & 2 \\
\hline L18 & 1.12991 & 0.00032 & 32.697 & 0.052 & 11.41 & 0.31 & 330.3 & 1.5 & 1 & 1 \\
\hline L19 & 1.13768 & 0.00025 & 34.339 & 0.073 & 5.49 & 0.02 & 59.8 & 0.1 & 1 & 1 \\
\hline L20 & 1.12429 & 0.00048 & 34.089 & 0.064 & 26.44 & 0.11 & 356.3 & 0.6 & 1 & 2 \\
\hline L21 & 1.13112 & 0.00055 & 36.179 & 0.069 & 11.67 & 0.03 & 763.3 & 3.1 & 1 & 1 \\
\hline L22 & 1.12005 & 0.00098 & 33.889 & 0.220 & 29.00 & 0.17 & 327.0 & 1.7 & 2 & 2 \\
\hline L23 & 1.11789 & 0.00038 & 34.183 & 0.171 & 27.33 & 0.43 & 325.0 & 1.0 & 2 & 2 \\
\hline L24 & 1.11896 & 0.00019 & 34.855 & 0.087 & 27.29 & 0.43 & 326.7 & 2.1 & 2 & 2 \\
\hline
\end{tabular}


Table 10: Parameter estimates, Wald and Likelihood Ratio (LR) test for the cone-jet response model.

\begin{tabular}{lcccc}
\hline \multirow{2}{*}{ Term } & Estimate & Standard error & \multicolumn{2}{c}{$p$-value } \\
& & & Wald test & LR test \\
\hline BSA & 5.1810408 & 3.8134782 & 0.1743 & 0.0519 \\
HP $\beta$ CD & 0.89532763 & 1.7500357 & 0.6089 & 0.5963 \\
PVP K25 & -0.0589736 & 1.6572741 & 0.9716 & 0.9716 \\
BSA * HP $\beta$ CD & -9.6248058 & 9.7810755 & 0.3251 & 0.2879 \\
BSA * PVP K25 & -13.561715 & 10.341393 & 0.1897 & 0.1365 \\
HP $\beta$ CD $*$ PVP K25 & 0.68869608 & 7.0188939 & 0.9218 & 0.9219 \\
\hline
\end{tabular}

Table 11: Parameter estimates, Wald and Likelihood Ratio (LR) test for the cone-jet response model including 3 additional confirmation points (L22, L23 and L24).

\begin{tabular}{lcccc}
\hline \multirow{2}{*}{ Term } & Estimate & Standard error & \multicolumn{2}{c}{$p$-value } \\
& & & Wald test & LR test \\
\hline BSA & 5.99074798 & 4.0235338 & 0.1365 & $0.0319^{*}$ \\
HP $\beta$ CD & 0.81006464 & 1.7376995 & 0.6411 & 0.6311 \\
PVP K25 & -0.284217 & 1.6713835 & 0.8650 & 0.8645 \\
BSA * HP $\beta$ CD & -10.539421 & 10.088892 & 0.2962 & 0.2591 \\
BSA * PVP K25 & -16.913351 & 10.381707 & 0.1033 & 0.0640 \\
HP $\beta$ CD $*$ PVP K25 & 1.93661982 & 6.9737515 & 0.7812 & 0.7813 \\
\hline
\end{tabular}




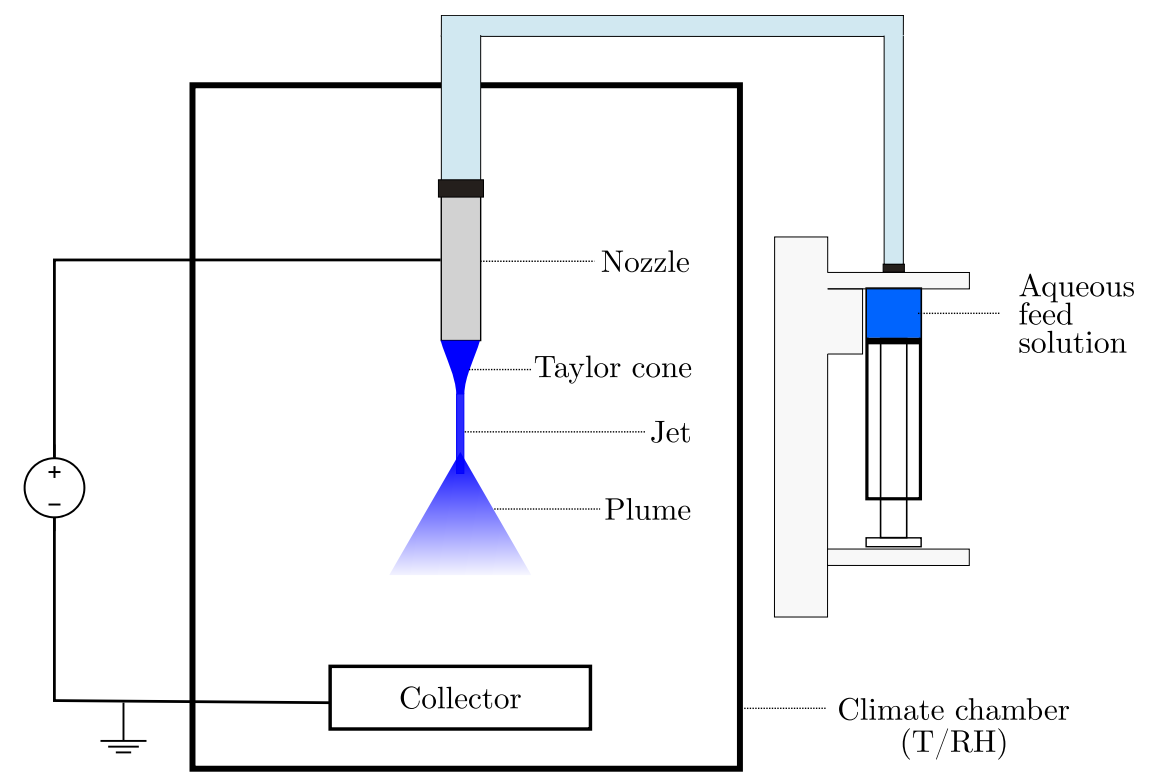

Figure 1: Schematic representation of the used electrospraying set-up.

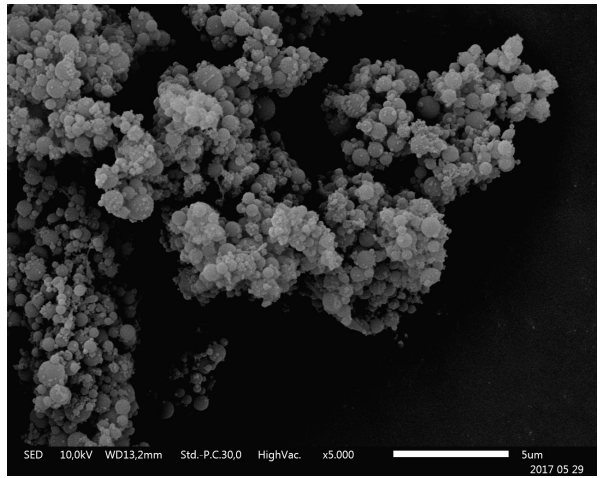

(a)

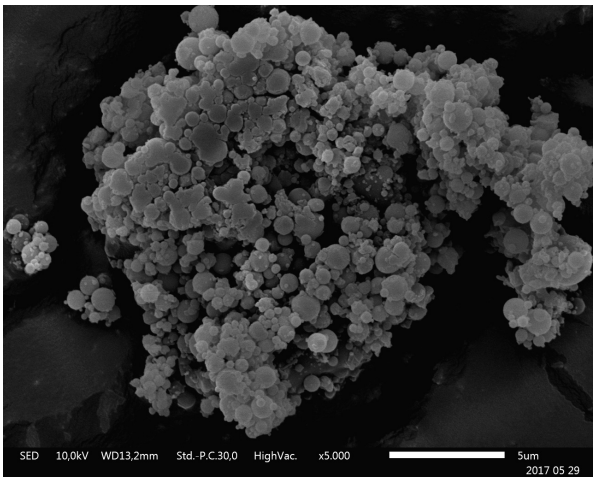

(b)

Figure 2: SEM images of the powder resulting from the $5 \% \mathrm{~m} / \mathrm{V}$ PVP K25 formulation (K25_5) given in Table 2. a depicts a representative SEM image of the particles collected from the visibly dry area of the powder bed, while b depicts a representative SEM image of the particles collected from the rest fraction of the powder bed where residual droplet could still be observed. 


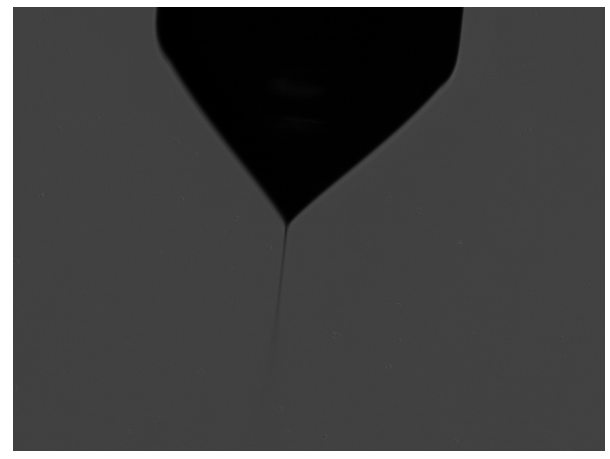

(a)

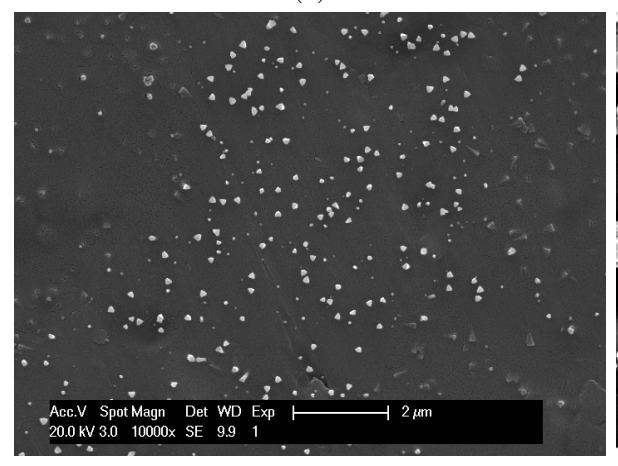

(c)

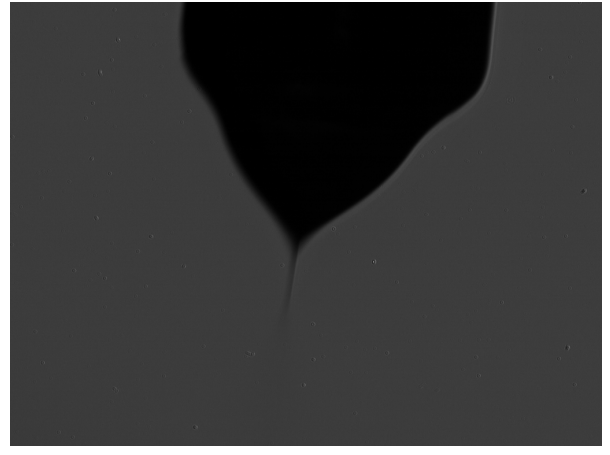

(b)

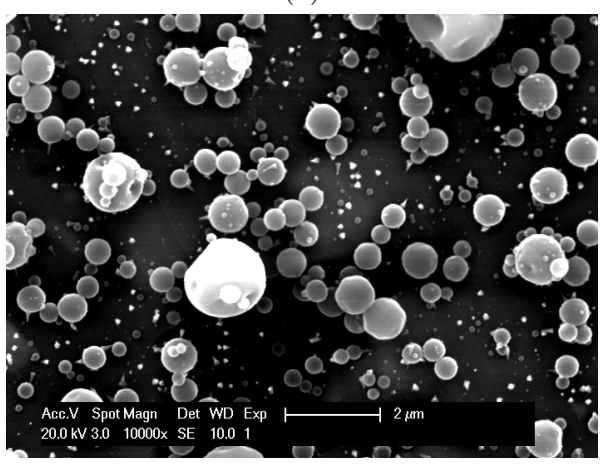

(d)

Figure 3: Images captured using a Fastcam SA2 high speed camera of the Taylor cone-jet mode observed during the electrospraying and SEM images of the resulting powder beds captured for formulations M2 (Figure 3a and Figure 3c, respectively), being a stable cone-jet, and formulation M14 (Figure 3b and Figure 3d, respectively), being an unstable cone-jet, of the mixture DoE shown in Table 3. 


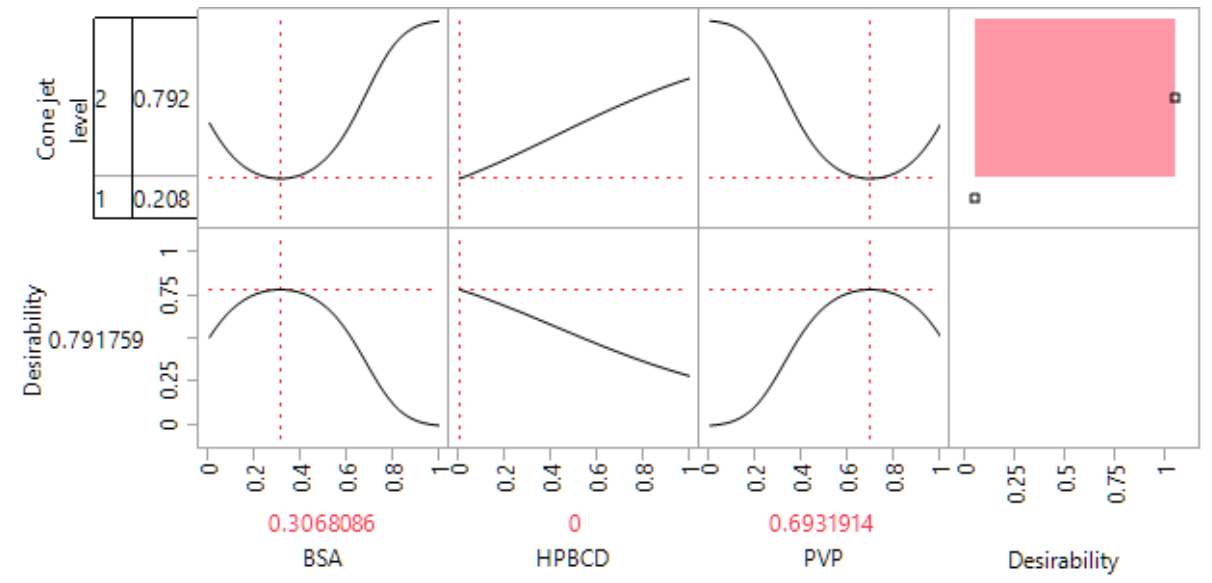

Figure 4: JMP prediction profiler and desirability function for the cone-jet response model.

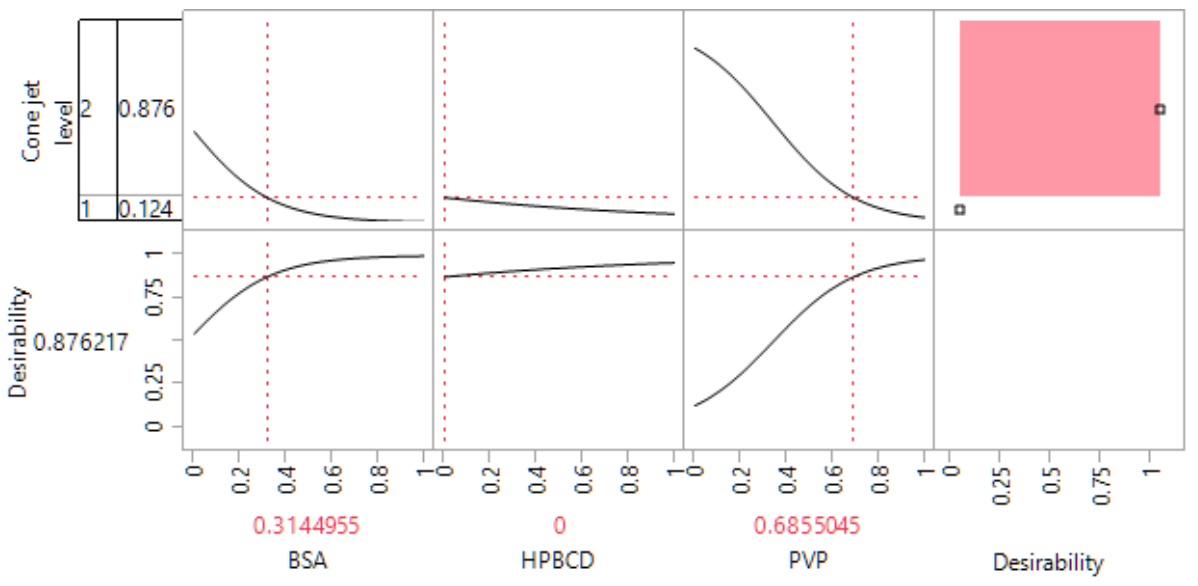

Figure 5: JMP prediction profiler and desirability function for the cone-jet response model including 3 additional confirmation points (L22, L23 and L24). 


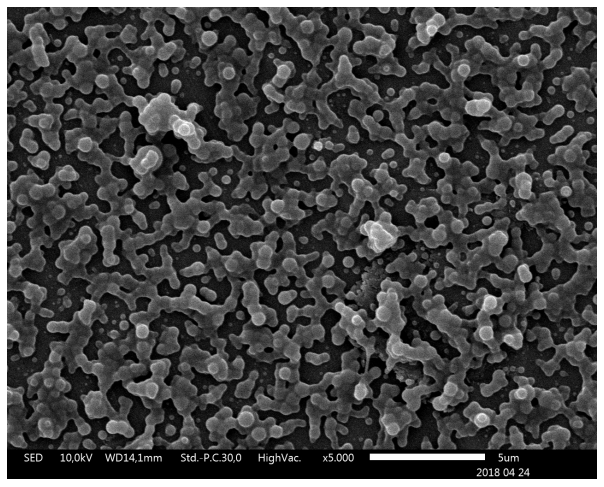

(a)

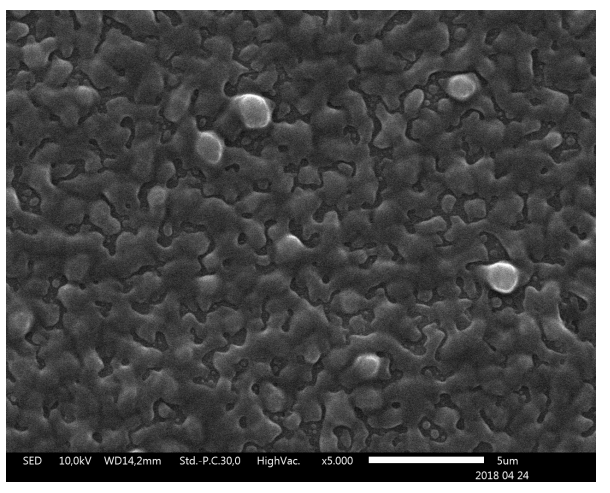

(b)

Figure 6: SEM images of the powders resulting from electrospraying formulations a L17 and b M14 from the mixture DoE following a simplex lattice (see Table 4). 